\title{
An Adaptive Variational Mode Decomposition Technique with Differential Evolution Algorithm and Its Application Analysis
}

\author{
Yuanxin Wang (iD \\ China Gezhouba Group Co., Ltd., Beijing 100022, China \\ Correspondence should be addressed to Yuanxin Wang; wangyuanxintdc@cggc.cn
}

Received 5 September 2021; Revised 23 September 2021; Accepted 27 October 2021; Published 11 November 2021

Academic Editor: Chaoqun Duan

Copyright ( $\odot 2021$ Yuanxin Wang. This is an open access article distributed under the Creative Commons Attribution License, which permits unrestricted use, distribution, and reproduction in any medium, provided the original work is properly cited.

\begin{abstract}
Variational mode decomposition is an adaptive nonrecursive signal decomposition and time-frequency distribution estimation method. The improper selection of the decomposition number will cause under decomposition or over decomposition, and the improper selection of the penalty factor will affect the bandwidth of modal components, so it is very necessary to look for the optimal parameter combination of the decomposition number and the penalty factor of variational mode decomposition. Hence, differential evolution algorithm is used to look for the optimization combination of the decomposition number and the penalty factor of variational mode decomposition because differential evolution algorithm has a good ability at global searching. The method is called adaptive variational mode decomposition technique with differential evolution algorithm. Application analysis and discussion of the adaptive variational mode decomposition technique with differential evolution algorithm are employed by combining with the experiment. The conclusions of the experiment are that the decomposition performance of the adaptive variational mode decomposition technique with differential evolution algorithm is better than that of variational mode decomposition.
\end{abstract}

\section{Introduction}

Variational mode decomposition is an adaptive nonrecursive signal decomposition and time-frequency distribution estimation method [1]. It can decompose the input signal into several intrinsic mode functions with bandwidth constraints and make each group of intrinsic mode function focus near different central frequencies, which has good noise robustness [2]. In recent years, variational mode decomposition has been applied to prediction field $[3,4]$. The improper selection of the decomposition number will cause under decomposition or over decomposition, and the improper selection of the penalty factor will affect the bandwidth of modal components; that is, the improper parameter combination of variational mode decomposition will influence the decomposition performance and the using effects in prediction field of variational mode decomposition. Hence, it is very necessary to look for the optimal parameter combination of the decomposition number and the penalty factor of variational mode decomposition.

Differential evolution algorithm is a population-based evolutionary optimization algorithm, which are motivated by the social behavior of animals [5-7]. Differential evolution algorithm is an efficient global optimization algorithm with simple structure, superior performance, and strong robustness, which can achieve a global optimal solution $[8,9]$. Hence, differential evolution algorithm is used to look for the optimization combination of the decomposition number and the penalty factor of variational mode decomposition, which can obtain the variational mode decomposition model with superior performance. The method is called adaptive variational mode decomposition technique with differential evolution algorithm. As the prediction for birth population is helpful for decision-making of related department, China's birth data are used to testify the decomposition performance of the adaptive variational mode 
decomposition technique with differential evolution algorithm, and the decomposition performance of the adaptive variational mode decomposition technique with differential evolution algorithm is judged by the prediction results for China's birth population.

\section{Adaptive Variational Mode Decomposition Technique with Differential Evolution Algorithm}

Variational mode decomposition is an adaptive nonrecursive signal decomposition and time-frequency distribution estimation method. It can decompose the input signal into several intrinsic mode functions with bandwidth constraints and make each group of intrinsic mode function focus near different central frequencies, which has good noise robustness. The basic idea of variational mode decomposition technique is to transform the signal decomposition process into a constraint variational problem [10].
In the variational mode decomposition technique, the constrained optimization problem can be described by the squared $L^{2}$ norm form:

$$
\begin{aligned}
& \min _{\left(u_{k}, \omega_{k}\right)}\left\{\sum_{k=1}^{K}\left\|\partial_{t}\left[\left(\sigma(t)+\frac{j}{\pi t}\right) * u_{k}(t)\right] e^{-j \omega_{k} t}\right\|_{2}^{2}\right\} \\
& \text { s.t. } \sum_{k=1}^{K} u_{k}(t)=s(t),
\end{aligned}
$$

where $K$ is the decomposition number, $\sigma(t)$ is the Dirac distribution, $s(t)$ is a signal with a set of time series data, $u_{k}(t)$ is an intrinsic mode function of $s(t), \omega_{k}$ is the center frequency of $u_{k}(t), \partial_{t}$ is the gradient operator, and $*$ is the convolution operator.

By introducing the penalty factor and Lagrangian multiplier, the constrained optimization problem can be converted into the mathematical formulation of an unconstrained optimization problem obtained with the induction of the augmented Lagrangian:

$$
L_{\left(u_{k}, \omega_{k}, r\right)}=\alpha \sum_{k=1}^{K}\left\|\partial_{t}\left[\left(\sigma(t)+\frac{j}{\pi t}\right) * u_{k}(t)\right] e^{-j \omega_{k} t}\right\|{ }_{2}^{2}+\left\|s(t)-\sum_{k=1}^{K} u_{k}(t)\right\|_{2}^{2}+\left\langle r(t), s(t)-\sum_{k=1}^{K} u_{k}(t)\right\rangle,
$$

where $\alpha$ is the penalty factor and $r(t)$ is the Lagrangian multiplier.

The multiplication operator alternating direction method is used to search the optimal solution of the above variational problem; the updated formulas of $u_{k}, \omega_{k}$, and $r$ are shown below:

$$
\begin{aligned}
U_{k}^{n+1}(\omega) & =\frac{S(\omega)-\sum_{i \neq k} U_{i}(\omega)+0.5 R(\omega)}{1+2 \alpha\left(\omega-\omega_{k}\right)^{2}}, \\
\omega_{k}^{n+1} & =\frac{\int_{0}^{\infty} \omega\left|U_{k}(\omega)\right|^{2} \mathrm{~d} \omega}{\int_{0}^{\infty}\left|U_{k}(\omega)\right|^{2} \mathrm{~d} \omega} \\
R^{n+1}(\omega) & =R^{n}(\omega)+\varepsilon\left(S(\omega)-\sum_{k=1}^{K} U_{k}^{n+1}(\omega)\right),
\end{aligned}
$$

where $S(\omega)$ is the Fourier transform signal of $s(t), R(\omega)$ is the Fourier transform signal of $r(t), U_{k}(\omega)$ is the Fourier transform signal of $u_{k}(t), n$ is the iterative times, and $\varepsilon$ is the noise tolerance coefficient.

Decomposition number $K$ and penalty factor $\alpha$ are two important parameters to determine whether variational mode decomposition can achieve good results. The improper selection of $K$ will cause under decomposition or over decomposition, and the improper selection of $\alpha$ will affect the bandwidth of modal components, so it is very necessary to look for the optimal parameter combination of $K$ and $\alpha$.
Differential evolution algorithm is a population-based evolutionary optimization algorithm, which are motivated by the social behavior of animals. Differential evolution algorithm is an efficient global optimization algorithm with simple structure, superior performance, and strong robustness, which can achieve a global optimal solution. Hence, differential evolution algorithm is used to look for the optimization combination of the parameters $K$ and $\alpha$ of variational mode decomposition. Differential evolution algorithm includes three operations: mutation operation, crossover operation, and selection operation $[11,12]$. In mutation operation, the mutation vector is generated by the mutation operator. Generate the recombinant population by the crossover operation. In selection operation, the better individual is selected to produce the next offspring according to the selection operator. The process of obtaining the optimization combination of the parameters $K$ and $\alpha$ of variational mode decomposition by differential evolution algorithm is as follows:

Step 1: as there are two parameters to be optimized, an initial population $\left\{X_{i}=\left(x_{i, 1}, x_{i, 2}\right) \mid i=1,2, \ldots, N\right\}$ is randomly generated in a given space according to equation (4), and the individual is composed of the parameters $K$ and $\alpha$ of variational mode decomposition:

$$
X_{i}=X_{i}^{\mathrm{low}}+\operatorname{rand} \cdot\left(X_{i}^{\mathrm{up}}-X_{i}^{\mathrm{low}}\right)
$$


where $X_{i}^{\text {low }}$ is the $i$ th individual's lower bound, $X_{i}^{\text {up }}$ is the $i$ th individual's upper bound, and rand is the random value with uniform distribution from 0 to 1 .

Step 2: average envelope entropy, shown in equation (5), is used as the objective function and computes the values of the objective function of the individuals:

$G_{(K, \alpha)}=\frac{1}{K} \sum_{k=1}^{K}\left[-\sum_{m=1}^{M}\left(\frac{c_{k}(m)}{\sum_{m=1}^{M} c_{k}(m)}\right) \log _{2}\left(\frac{c_{k}(m)}{\sum_{m=1}^{M} c_{k}(m)}\right)\right]$,

where $c_{k}(m)$ is the $k$ th intrinsic mode function's envelope entropy and $M$ is the sampling numbers.

The optimization problem of the combination of the parameters $K$ and $\alpha$ of variational mode decomposition is expressed as $\min \left\{G_{(K, \alpha)}\right\}$. The smaller the value of the objective function of the individual is, the better the combination of the parameters $K$ and $\alpha$ of variational mode decomposition is.

Step 3: generate the mutated individuals with three different random individuals according to

$$
y_{i, j}(t+1)=x_{z 1, j}(t)+F_{i}(t)\left[x_{z 2, j}(t)-x_{z 3, j}(t)\right],
$$

where $F_{i}(t)$ is the $i$ th individual's scaling factor, $x_{z 1, j}(t), x_{z 2, j}(t)$, and $x_{z 3, j}(t)$ are three different individuals, and $i \neq z 1 \neq z 2 \neq z 3$.

Step 4: generate the recombinant population by the crossover operation, if rand is less than or equal to $\mathrm{CR}$ or $j$ is equal to $j_{\text {rand }}, u_{i, j}(t)=y_{i, j}(t)$, otherwise, $u_{i, j}(t)=x_{i, j}(t)$, where CR is the crossover rate and $j_{\text {rand }}$ is the integer randomly chosen from 1 to 2 .

Step 5: the individual with smaller value of the objective function is selected to produce the next offspring according to the selection operator.

Step 6: repeat the evolutionary cycle until the maximum number of iterations is reached. Otherwise, go to Step 2. Then, the optimal combination of the parameters $K$ and $\alpha$ of variational mode decomposition is obtained.

\section{Application Analysis and Discussion of the Adaptive Variational Mode Decomposition Technique with Differential Evolution Algorithm}

The number of births has a great influence on society and economy of a country; the prediction for birth population is helpful for decision-making of related department. Hence, China's births data are used to testify the decomposition performance of the adaptive variational mode decomposition technique with differential evolution algorithm. The optimal combination of the parameters $K$ and $\alpha$ is obtained by differential evolution algorithm, and the adaptive variational mode decomposition model with differential evolution algorithm is realized to obtain the variational mode decomposition model with superior performance. China's birth data are decomposed to form several intrinsic mode

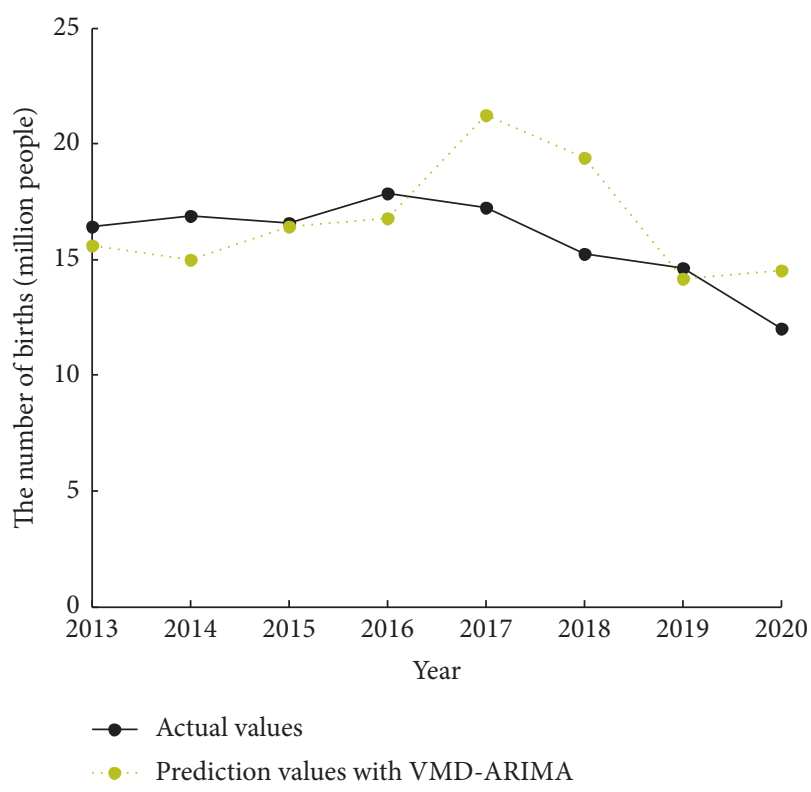

FIgURE 1: The actual China's birth population and the prediction results for China's birth population with VMD-ARIMA.

functions by using the adaptive variational mode decomposition technique with differential evolution algorithm.

Autoregressive integrated moving average model is used to predict the several intrinsic mode functions of China's birth population; autoregressive integrated moving average is a simple and practical prediction method, which reduces the complexity of the model and is suitable for the prediction problem of small samples [13-15]. The intrinsic mode functions of China's birth population have an important influence on the prediction results for China's birth population, so the decomposition performance of the adaptive variational mode decomposition technique with differential evolution algorithm can be judged by the prediction results for China's birth population.

The actual China's birth population and the prediction results for China's birth population with variational mode decomposition-autoregressive integrated moving average (VMD-ARIMA) are given in Figure 1, and the actual China's birth population and the prediction results for China's birth population with adaptive variational mode decomposition technique with differential evolution algorithm-autoregressive integrated moving average (DAVMD-ARIMA) are given in Figure 2. By the trend analysis of the prediction results for China's birth population with DAVMD-ARIMA, China's birth population takes on decreasing trend in recent years, which is in accord with actual situation. The trend of the prediction results for China's birth population with DAVMD-ARIMA is near to the trend of the actual China's birth population than the trend of the prediction results for China's birth population with VMD-ARIMA.

According to the relative errors for China's birth population prediction of VMD-ARIMA and DAVMD-ARIMA given in Figure 3, the average relative errors for China's birth population prediction of VMD-ARIMA and DAVMDARIMA are calculated. The average relative error for China's 


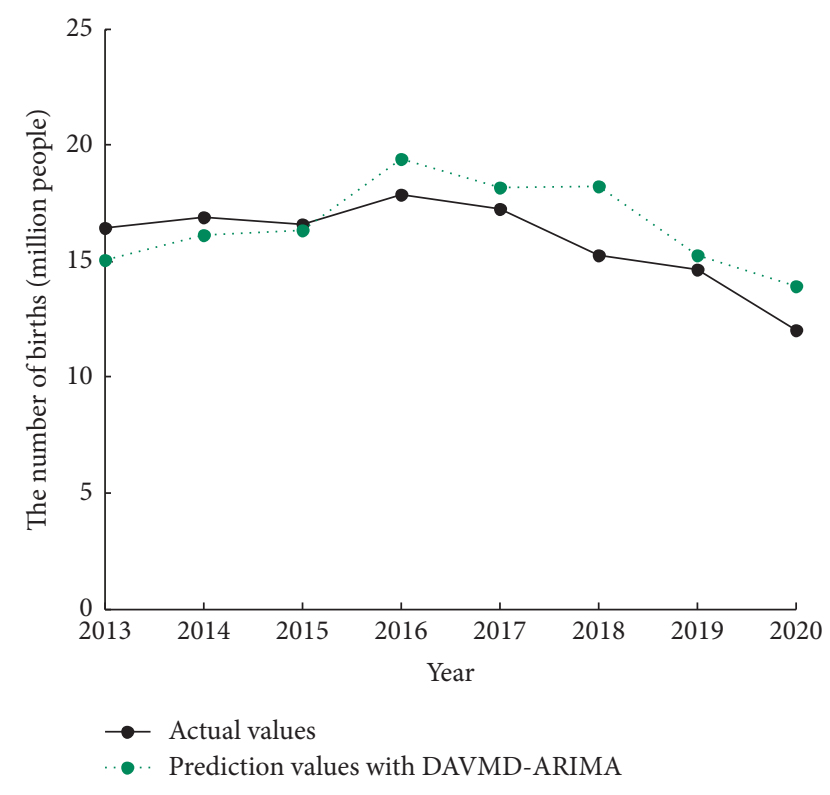

FIgURE 2: The actual China's birth population and the prediction results for China's birth population with DAVMD-ARIMA.

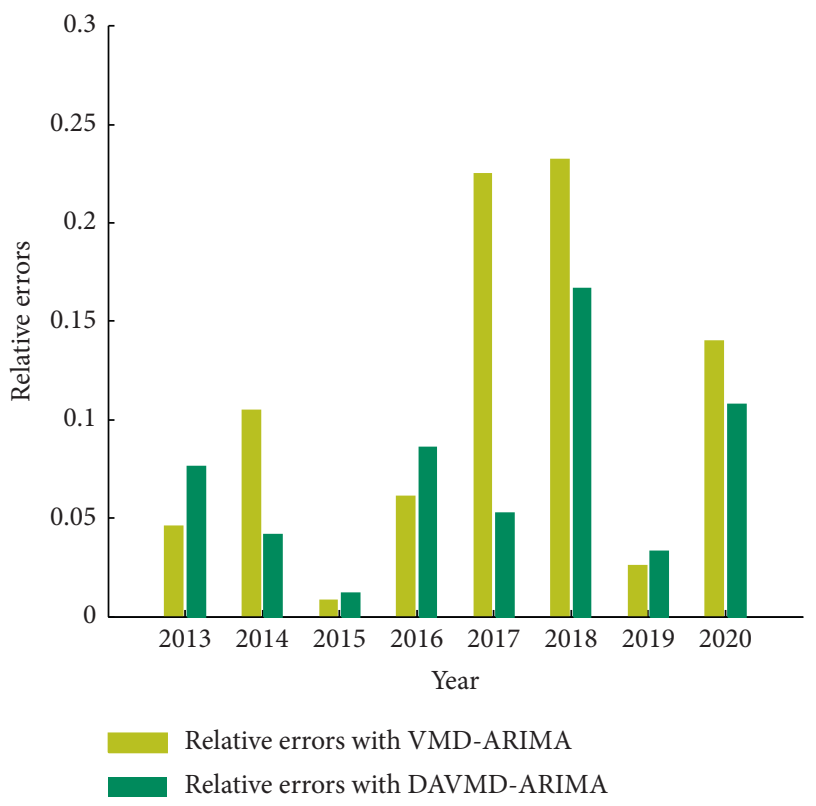

Figure 3: The relative errors for China's birth population prediction of VMD-ARIMA and DAVMD-ARIMA.

birth population prediction with VMD-ARIMA is $10.52 \%$; however, the average relative error for China's birth population prediction with DAVMD-ARIMA is $7.2 \%$; that is, the average relative error for China's birth population prediction with DAVMD is smaller than the average relative error for China's birth population prediction with VMDARIMA, so DAVMD-ARIMA is more suitable for China's birth population prediction than VMD-ARIMA. Accordingly, the decomposition performance of the adaptive variational mode decomposition technique with differential evolution algorithm is better than that of variational mode decomposition.

\section{Conclusions}

In order to solve the selection problem of the parameter combination of the decomposition number and the penalty factor of variational mode decomposition, an adaptive variational mode decomposition technique with differential evolution algorithm is proposed to obtain the variational mode decomposition model with superior performance. As the prediction for birth population is helpful for decisionmaking of related department, the decomposition performance of the adaptive variational mode decomposition technique with differential evolution algorithm is judged by the prediction results for China's birth population. The results are as follows:

(1) By the trend analysis of the prediction results for China's birth population with DAVMD-ARIMA, China's birth population takes on decreasing trend in recent years, which is in accord with actual situation. The trend of the prediction results for China's birth population with DAVMD-ARIMA is near to the trend of the actual China's birth population than the trend of the prediction results for China's birth population with VMD-ARIMA.

(2) The average relative error for China's birth population prediction with DAVMD-ARIMA is smaller than the average relative error for China's birth population prediction with VMD-ARIMA.

The conclusions are as follows:

(1) DAVMD-ARIMA is more suitable for China's birth population prediction than VMD-ARIMA

(2) Differential evolution algorithm can find the optimization combination of the decomposition number and the penalty factor of variational mode decomposition; then, the decomposition performance of the adaptive variational mode decomposition technique with differential evolution algorithm is better than that of variational mode decomposition

\section{Data Availability}

The data used to support the findings of the study are available from the author upon request.

\section{Conflicts of Interest}

The author declares no conflicts of interest with respect to the publication of this article.

\section{References}

[1] I. C. Yadav, S. Shahnawazuddin, and G. Pradhan, "Addressing noise and pitch sensitivity of speech recognition system through variational mode decomposition based spectral smoothing," Digital Signal Processing, vol. 86, pp. 55-64, 2019.

[2] H. Sharma, "Heart rate extraction from PPG signals using variational mode decomposition," Biocybernetics and Biomedical Engineering, vol. 39, no. 1, pp. 75-86, 2019. 
[3] B. Karan and S. Sekhar Sahu, "An improved framework for Parkinson's disease prediction using Variational Mode Decomposition-Hilbert spectrum of speech signal," Biocybernetics and Biomedical Engineering, vol. 41, no. 2, pp. 717-732, 2021.

[4] I. Majumder, P. K. Dash, and R. Bisoi, "Variational mode decomposition based low rank robust kernel extreme learning machine for solar irradiation forecasting," Energy Conversion and Management, vol. 171, pp. 787-806, 2018.

[5] P. Chiradeja, S. Yoomak, and A. Ngaopitakkul, "Optimal allocation of multi-DG on distribution system reliability and power losses using differential evolution algorithm," Energy Procedia, vol. 141, pp. 512-516, 2017.

[6] W.-S. Lee, Yi-T. Chen, and Y. Kao, "Optimal chiller loading by differential evolution algorithm for reducing energy consumption," Energy and Buildings, vol. 43, no. 2-3, pp. 599-604, 2011.

[7] D. Chen, X. Zhang, Y. Xie, and H. Ding, "Precise estimation of cutting force coefficients and cutter runout in milling using differential evolution algorithm," Procedia CIRP, vol. 77, pp. 283-286, 2018.

[8] S. Mete, S. Ozer, and H. Zorlu, "System identification using Hammerstein model optimized with differential evolution algorithm," AEU-International Journal of Electronics and Communications, vol. 70, no. 12, pp. 1667-1675, 2016.

[9] S. Jazebi, S. H. Hosseinian, and B. Vahidi, "DSTATCOM allocation in distribution networks considering reconfiguration using differential evolution algorithm," Energy Conversion and Management, vol. 52, no. 7, pp. 2777-2783, 2011.

[10] A. Bagheri, O. E. Ozbulut, and D. K. Harris, "Structural system identification based on variational mode decomposition," Journal of Sound and Vibration, vol. 417, pp. 182-197, 2018.

[11] H. Zorlu, "Optimization of weighted myriad filters with differential evolution algorithm," AEU-International Journal of Electronics and Communications, vol. 77, pp. 1-9, 2017.

[12] K. Nandhini and S. R. Balasundaram, "Extracting easy to understand summary using differential evolution algorithm," Swarm and Evolutionary Computation, vol. 16, pp. 19-27, 2014.

[13] H.-K. Yu, N. Y. Kim, S. S. Kim, C. Chu, and M. K. Kee, "Forecasting the number of human immunodeficiency virus infections in the Korean population using the autoregressive integrated moving average model," Osong Public Health and Research Perspectives, vol. 4, no. 6, pp. 358-362, 2013.

[14] R. $\mathrm{Wu}$, "Least absolute deviation estimation for general fractionally integrated autoregressive moving average time series models," Statistics \& Probability Letters, vol. 94, pp. 69-76, 2014.

[15] Yi-S. Lee and L.-I. Tong, "Forecasting time series using a methodology based on autoregressive integrated moving average and genetic programming," Knowledge-Based Systems, vol. 24, no. 1, pp. 66-72, 2011. 